\title{
Treatment of Osteitis Pubis in Non-Athlete Female Patients
}

\author{
E. Kavroudakis, P.K. Karampinas, D.S. Evangelopoulos ${ }^{*}$ and J. Vlamis \\ $3^{\text {rd }}$ Department of Orthopaedics, University of Athens, Greece
}

\begin{abstract}
Background: Osteitis pubis represents a non-infectious inflammation of the pubic symphysis causing varying degrees of lower abdominal and pelvic pain. Although, the disease is believed to affect mainly young athletic patients, it is also encountered in other specific patient groups. Both conservative and surgical treatment options are available. While for elite athletes surgical treatment is indicated, leading to fast pain relief and mobilization, for non-athletic patients no clear indication can be established.
\end{abstract}

Methods: Eight non-athletic women with osteitis pubis, referred to our Department for treatment, were evaluated. All were initially treated conservatively with bed rest, per os non steroidal anti-inflammatory drugs and physical therapy.

Results: Seven patients improved significantly with conservative treatment while one displayed no improvement and was treated surgically with arthrodesis.

Conclusion: We conclude that, for non-athletic female patients suffering from osteitis pubis, surgery is rarely required and that conservative treatment by means of non steroidal anti inflammatory drugs and physical modalities represents a fair option regarding pain and limitation of everyday activity.

Keywords: Osteitis pubis, pubic osteomyelitis, conservative treatment.

\section{INTRODUCTION}

Osteitis pubis has been originally described in patients' following suprapubic surgery for urological or gynecological pathologic modalities and remains a well-known complication of invasive procedures of the pelvic area [1]. Although it represents a common cause of pain in athletes, particularly soccer players, it has often been reported after trauma, rheumatic disorders, pregnancy and parturition [2-4, 6]. It can affect all age groups; however it is rarely encountered in the pediatric population. Literature data reports that it occurs most commonly in men aged between 30 to 50 years. Women are affected more in their mid $30 \mathrm{~s}$ [3].

Nowadays, the disease is regarded as a non-infectious inflammation of the pubic symphysis leading to varying degrees of lower abdominal and pelvic pain. Pain is localized over the pubic symphysis and may radiate to the groin, medial thigh, or abdomen [3]. Differential diagnosis mandates special attention since the disease has to be distinguished from pubic osteomyelitis $[2,3,5]$.

Both conservative and surgical treatment options are available. Treatment choice depends on patient's characteristics. For elite athletes, surgical treatment shows good results and permits fast pain relief and mobilization [2, 3]. However, for non-athletic patients, no definite conclusion can be drawn. This is mainly due to the rarity of the disease and the limited size of the study groups.

*Address correspondence to this author at 48 lemesou str 15669 , Athens Greece; Tel: 00302106280890; E-mail: ds.evangelopoulos@gmail.com
The aim of this study is to determine the treatment results for osteitis pubis in a non-athletic study group at our institution and to review these results with those already reported in the literature.

\section{METHODS}

From 1999 to 2006, 17 non-athletic female patients were treated at the authors' institution for pain over the symphysis pubis. On admission, all patients had a detailed medical history and thorough physical and laboratory examination. Differential diagnosis included pubic osteomyelitis, stress fracture, groin strain, pelvic fracture, muscle contusions, tendon injuries, acetabular labral tears, bursitis, rheumatologic disorders and chronic symphyseal injury.

After appropriate laboratory and clinical diagnostic methods, osteomyelitis of the symphysis pubis was diagnosed in 6 patients and pelvic fracture in 3 patients. All the aforementioned cases were excluded from our study. The eight remaining patients who displayed no clinical or laboratory findings were diagnosed to suffer of osteitis pubis and were included in the study group. Their mean age was 43,1 years (range from 36 to 52 years). All of them had given birth to two or more children. Four of them had undergone three spontaneous deliveries, two had four spontaneous deliveries and two had two deliveries with caesarean section.

All patients received conservative treatment and were followed up for a mean period of 24 months. Five women complained of pain directly above the symphysis pubis and one for anterior right hip pain. Two patients had already been examined by a general surgeon for lower abdominal 
pain. None of the subjects had undergone any lower abdominal surgical, urologic or gynecologic operation in the past 10 years.

\section{RESULTS}

The onset of symptoms varied from three weeks to three and a half years before seeking medical attention (mean 16,8 months). In five patients, an inciting event could be recollected. Three patients reported a fall from low altitude while two a low force kicks. The remaining patients described a gradual onset of symptoms. During the physical examination, all patients demonstrated pubic pain or tenderness as well as a waddling gait and pain on hip abduction. In two patients, crepitus could be felt by pressing against the pubic symphysis.

The white blood cell count was slightly elevated (average $10,800 \times 10^{3} / \mu \mathrm{L}$ ) in all but one patients but with a normal ratio between the different types of white blood cells. The erythrocyte sedimentation rate was also slightly elevated, ranging from 27 to $48 \mathrm{~mm} / \mathrm{h}$ (mean $38,2 \mathrm{~mm} / \mathrm{h}$ ). Blood biochemistry examination did not reveal any specific abnormality. Serologic examination revealed normal levels of C-reactive protein.

All 8 patients had a plain antero-posterior X-ray examination of the pelvis. In three patients early radiographic signs of Osteitis pubis were observed with rarefaction of the adjacent pubic bones and widening of the symphysis pubis. Four patients demonstrated late radiologic signs of the disease with sclerosis and narrowing of the symphyseal joint space. These findings were also verified by computerized tomography and magnetic resonance imaging.

Initially, all patients were treated conservatively (Fig. 1). Bed rest was implemented for 4 to 6 days followed by progressive ambulation with the aid of an assistive device such as a cane or crutches. All patients were advised to avoid activities that might stress the pelvic ring. Modalities such as heat or ice were suggested and were used according to individual preference (5 out of 8 patients reported some symptomatic relief with locally applied ice and 2 with heat).

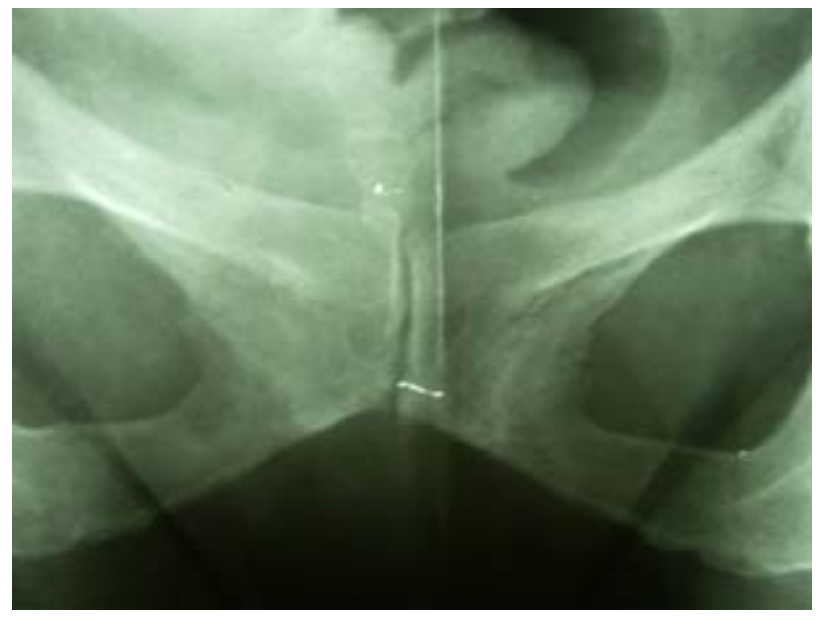

Fig. (1). X-rays of osteitis pubis in two patients treated conservatively.
Every patient was regularly re-examined at intervals ranging from 10 to 14 days during the first 2 months and then every one or two months. During the first four weeks of treatment, 6 patients improved markedly. At the two month follow up, all patients were encouraged to follow a supervised program for strengthening the abdominal and hip muscles and improving range of motion of the hip, particularly the muscles of internal rotation. Non steroidal anti-inflammatory agents were prescribed invariably in all patients provided that there was no contraindication. Local antiflammatory injections were administered to achieve fast pain relief. Five patients reached a completely pain-free state after 9 months and did not relapse during the follow-up. Two reported reoccurrence of pain after intense physical activity. One failed to respond to any conservative treatment, displaying severely limited functional activity (Fig. 2). She was submitted to pubic symphysiodesis eight months after her referral to our department (Fig. 3). One month post stabilization, the pain was markedly reduced and she had very mild limitation of her daily functional activity

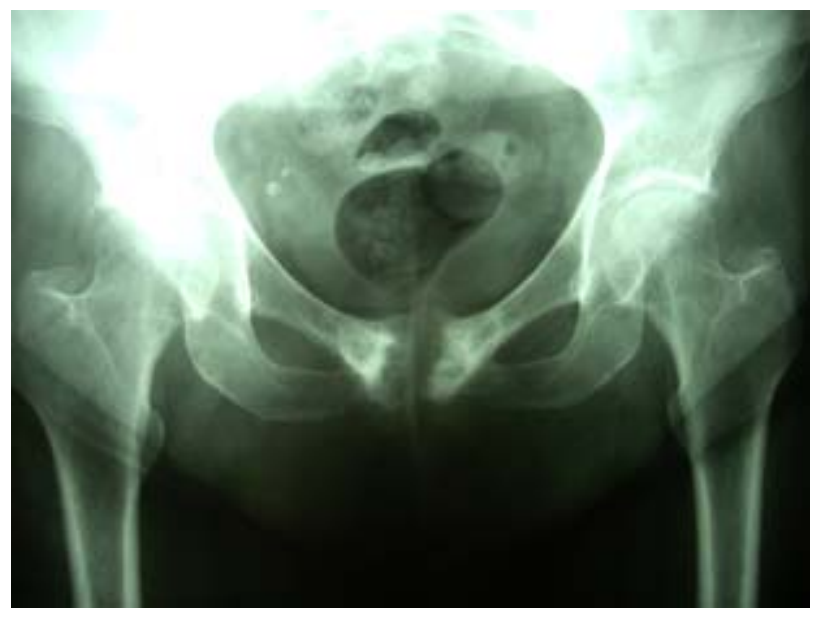

Fig. (2). Pre operative x-ray of osteitis pubis in a patient treated with internal fixation.

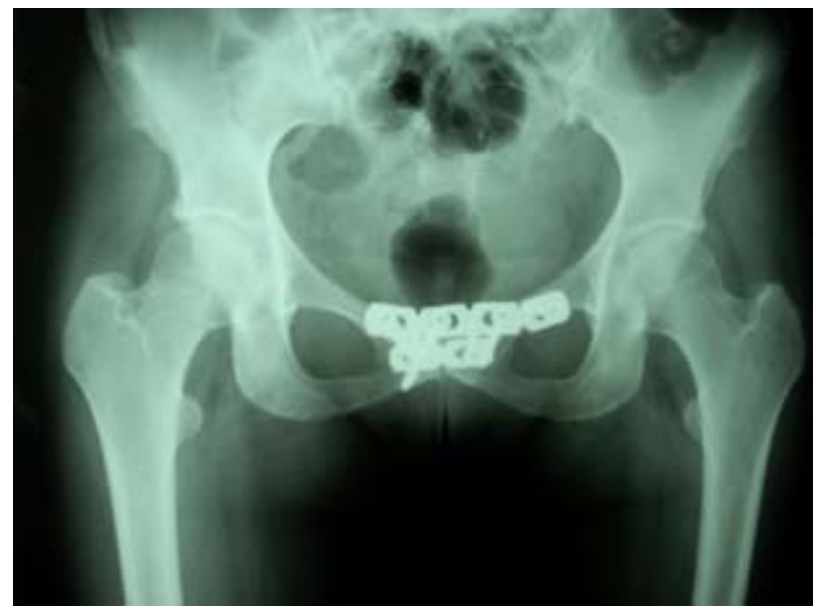

Fig. (3). Post operative $x$-ray in a patient treated with internal fixation (simfisiodesis). 


\section{DISCUSSION}

Osteitis pubis represents a well known pathologic modality. The disease usually affects young athletes and is characterized by excessive strains on the pubic bone due to rigorous demands of competitive sport $[3,4,6]$. Its incidence in the general athletic population is estimated to $0.5 \%-7 \%$ [7-9]. Although rare in non-athletic populations, the presence of intense muscle forces acting on the symphysis pubis may lead to the development of similar symptoms. Gonik et al. reported a case of postpartum osteitis pubis while Scott et al, in their study, announced a possible association between the disease and previous pregnancy $[10,11]$. A possible mechanism is that the hormone-induced ligamentous laxity may facilitate increased motion and impaction of the articular surfaces of the symphysis [12]. Osteitis pubis has also been shown in the population of rheumatic patients due to impaired circulation, leading to thrombosis and circulatory obstruction of the venous plexus supplying the symphysis [12]. Scott et al. reported its presence on patients suffering from ankylosing spondylitis [11]. Additionally, SierraJimenez et al, assessing the prevalence of selected clinical and radiological features of tendinous and ligamentous derangements in a consecutive sample of patients with systemic lupus erythematosus, announced that $6 \%$ of the population suffered of osteitis pubis [13].

The disease's symptoms may mimic other pathologic modalities affecting pubic symphysis [2, 3, 5, 14, 15]. Since pubic osteomyelitis is one of those modalities, the clinician must maintain a high index of suspicion. Typical features of pubic symphysis infection include fever, pubic pain, painful or waddling gait, pain with hip motion, and groin pain [16]. Radiologically, osteomyelitis is characterized by the development of infiltrates, cortical involvement and local osteopenia. Apart from plain radiograms, bone scans, CTscans and magnetic resonance imaging (MRI) may be required to assist the differential diagnosis [15]. If necessary, bone cultures after puncture may be required to set the diagnosis.

Different surgical techniques have been described in the literature, including curettage, arthrodesis, wedge resection, wide resection as well as endoscopic pubic symphysiectomy $[12,17]$. Athletes seem to respond well to surgical treatment. In their study on 23 athletes treated with curettage for osteitis pubis, Radic et al. announced that $61 \%$ demonstrated no pain and full activity [18]. Treating surgically elite level male athletes, Paajanen et al, reported very good results by introducing a polypropylene mesh into the preperitoneal retropubic space through a minimal invasive approach [19]. Similar conclusions are also reported by Mehim et al. [12]. However, the last authors indicate that surgery should not be the treatment of choice for other subpopulations. Analyzing their results, they reported an unpredictable outcome for the surgical treatment of postpartum and rheumatologic osteitis pubis [12]. The data of the present study demonstrates that for non athletic female patients, surgery is rarely required. Most of the patients of this study group demonstrated good results with conservative treatment and only one required a surgical intervention. For this patient, after thorough debridement of the fibrocartilaginous disc and hyaline cartilage, two $3.5-\mathrm{mm}$ plates were orthogonally applied to stabilize the symphysis. Mehin et al, in his study on surgical treatment for osteitis pubis, reported different fiaxation techniques either with single $3.5-\mathrm{mm} / 4.5 \mathrm{~mm}$ plates or with the combination of a $4.5 \mathrm{~mm}$ and a $3.5 \mathrm{~mm}$ plates (one on the superior and the other on the anterior surfaceof the symphysis) [12, 20, 21]. Similar conclusions are reported by other studies in the literature $[1,4,12]$. Additionally, no specific factor could be isolated to predict the need for surgical treatment. It is therefore assumed that personality traits could have a role as well.

The results show that, for non-athetic women suffering from osteitis pubis, conservative treatment with the help of non steroidal anti inflammatory drugs and physical modalities, offers very good results regarding pain and limitation of everyday activity.

However, this study possesses certain limitations. It is retrospective and also refers to a limited number of patients. Taking into account the rarity of the disease in the group under investigation, the results can be considered relatively reliable but further investigations are needed to determine the role, timing and method of a possible surgical approach in this type of patients.

\section{ACKNOWLEDGEMENT}

None declared.

\section{CONFLICT OF INTERESTS}

'The author(s) declare that they have no competing interests'.

\section{REFERENCES}

[1] Andrews SK, Carek PJ. Osteitis Pubis: A Diagnosis for the Family Physician. J Am Board Fam Pract 1998; 11(4): 291-5

[2] Fricker PA, Taunton JE, Ammann W. Osteitis pubis in athletes. Infection, inflammation or injury? Sports Med 1991; 12(4): 266-79.

[3] Johnson R. Osteitis pubis. Curr Sports Med Rep 2003; 2(2): 98102.

[4] Vincent C. Osteitis pubis. J Am Board Fam Pract 1993; 6(5): 492-6

[5] Knoeller SM, Uhl M, Herget GW. Osteitis or osteomyelitis of the pubis? A diagnostic and therapeutic challenge: report of 9 cases and review of the literature. Acta Orthop Belg 2006; 72(5): 541-8.

[6] Grace JN, Sim FH, Shives TC, Coventry MB. Wedge resection of the symphysis pubis for the treatment of osteitis pubis. J Bone Joint Surg Am 1989; 71(3): 358-64.

[7] Lovell G. The diagnosis of chronic groin pain in athletes: a review of 189 cases. Aust J Sci Med Sport 1995; 27(3): 76-9.

[8] Dahan R. Rehabilitation of muscle-tendon injuries to the hip, pelvis and groin areas. Sports Med Arthrosc Rev 1997; 5: 326-33.

[9] Westlin N. Groin pain in athletes from southern Sweden. Sports Med Arthrosc Rev 1997; 5: 280-84.

[10] Gonik B, Stringer CA. Postpartum osteitis pubis. South Med J 1985; 78(2): 213-4.

[11] Scott DL, Eastmond CJ, Wright V. A comparative radiological study of the pubic symphysis in rheumatic disorders. Ann Rheum Dis 1979; 38(6): 529-34.

[12] Mehin R, Meek R, O'Brien P, Blachut P. Surgery for osteitis pubis. Can J Surg 2006; 49(3): 170-6.

[13] Sierra-Jimenez G, Sanchez-Ortiz A, Aceves-Avila FJ, HernandezRios G, Durán-Barragán S, Ramos-Remus C. Tendinous and ligamentous derangements in systemic lupus erythematosus. J Rheumatol 2008; 35(11): 2187-91

[14] Bali K, Kumar V, Patel S, Mootha AK. Tuberculosis of symphysis pubis in a 17 year old male: a rare case presentation and review of literature. J Orthop Surg Res 2010; 5: 63.

[15] Zoga AC, Kavanagh EC, Omar IM, et al. Athletic pubalgia and the "sports hernia": MR imaging findings. Radiology 2008; 247(3): 797-807.

[16] Ross JJ, Hu LT. Septic arthritis of the pubic symphysis: review of 100 cases. Medicine (Baltimore) 2003; 82: 340-5. 
[17] Matsuda DK. Endoscopic pubic symphysectomy for reclacitrant osteitis pubis associated with bilateral femoroacetabular impingement. Orthopedics 2010; 10: 199-203.

[18] Radic R, Annear P. Use of pubic symphysis curettage for treatment-resistant osteitis pubis in athletes. Am J Sports Med 2008; 36(1): 122-8.

[19] Paajanen H, Heikkinen J, Hermunen H, Airo I. Successful treatment of osteitis pubis by using totally extraperitoneal endoscopic technique. Int J Sports Med 2005; 26(4): 303-6.
[20] Matta JM. Indications for anterior fixation of pelvic fractures. Clin Orthop Relat Res 1996; 329: 88-96.

[21] Hearn TC, Willet K, Schopfer A, et al. Mechanical response of the intact, disrupted and internally fixed pelvic ring to stance-related loading. In: Surgery of the Pelvis and Acetabulum: An International Consensus, Final Program and Syllabus; Pittsburgh, PA $1992 ; 86$

Received: April 23, 2011

Revised: July 26, 2011

Accepted: July 28, 2011

(C) Kavroudakis et al.; Licensee Bentham Open.

This is an open access article licensed under the terms of the Creative Commons Attribution Non-Commercial License (http: //creativecommons.org/licenses/by-nc/3.0/) which permits unrestricted, non-commercial use, distribution and reproduction in any medium, provided the work is properly cited. 